\title{
Highly Stable Perovskite Supercrystals via Oil-in-Oil Templating
}

Tang, Yingying; Gomez, Leyre; Lesage, Arnon; Marino, Emanuele; Kodger, Thomas E.; Meijer, Janne Mieke; Kolpakov, Paul; Meng, Jie; Zheng, Kaibo; Gregorkiewicz, Tom

Total number of authors:

11

Published in:

Nano Letters

Link to article, DOI:

10.1021/acs.nanolett.0c02005

Publication date:

2020

Document Version

Publisher's PDF, also known as Version of record

Link back to DTU Orbit

Citation (APA):

Tang, Y., Gomez, L., Lesage, A., Marino, E., Kodger, T. E., Meijer, J. M., Kolpakov, P., Meng, J., Zheng, K., Gregorkiewicz, T., \& Schall, P. (2020). Highly Stable Perovskite Supercrystals via Oil-in-Oil Templating. Nano Letters, 20(8), 5997-6004. https://doi.org/10.1021/acs.nanolett.0c02005

\section{General rights}

Copyright and moral rights for the publications made accessible in the public portal are retained by the authors and/or other copyright owners and it is a condition of accessing publications that users recognise and abide by the legal requirements associated with these rights.

- Users may download and print one copy of any publication from the public portal for the purpose of private study or research.

- You may not further distribute the material or use it for any profit-making activity or commercial gain

- You may freely distribute the URL identifying the publication in the public portal 


\section{Highly Stable Perovskite Supercrystals via Oil-in-Oil Templating}

Yingying Tang, Leyre Gomez, Arnon Lesage, Emanuele Marino, Thomas E. Kodger, Janne-Mieke Meijer, Paul Kolpakov, Jie Meng, Kaibo Zheng, Tom Gregorkiewicz, and Peter Schall*

Cite This: Nano Lett. 2020, 20, 5997-6004

Read Online

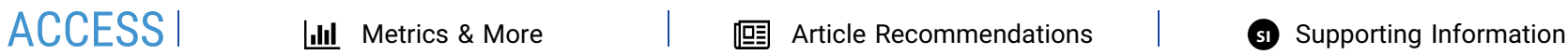

ABSTRACT: Inorganic perovskites display an enticing foreground for their wide range of optoelectronic applications. Recently, supercrystals (SCs) of inorganic perovskite nanocrystals (NCs) have been reported to possess highly ordered structure as well as novel collective optical properties, opening new opportunities for efficient films. Here, we report the large-scale assembly control of spherical, cubic, and hexagonal SCs of inorganic perovskite NCs through templating by oil-in-oil emulsions. We show that an interplay between the roundness of the cubic NCs and the tension of the confining droplet surface sets the superstructure morphology, and we exploit this interplay to design dense hyperlattices of SCs. The SC films show strongly enhanced stability for at least two months without obvious structural degradation and minor optical changes. Our results on the controlled large-scale assembly of perovskite NC superstructures provide new prospects for the bottom-up production of optoelectronic devices based on the microfluidic production of mesoscopic building blocks.

KEYWORDS: assembly, emulsion-droplet templating, supercrystals, perovskite films, stability

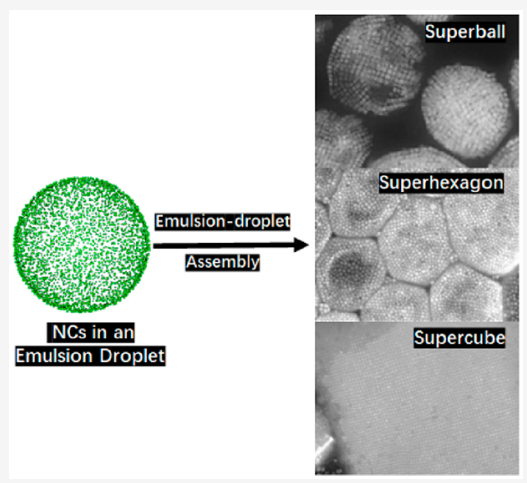

T hree-dimensional (3D) highly ordered supercrystals (SCs) assembled from individual nanocrystals (NCs) promise the advent of novel optoelectronic devices based on "artificial solids", exhibiting tunable collective solid-like properties from assembled semiconductor NCs, where the individual NCs can be regarded as artificial atoms. ${ }^{1}$ The length scale and crystallographic orientation of the ordered arrangements of the NCs can then be controlled by either physical or chemical methods. After self-assembly, the SCs usually display novel collective properties, that is, superfluorescence, ${ }^{2}$ bathochromic shift, ${ }^{3}$ and minibands, ${ }^{4}$ prompted by their special configuration and periodicity. Their self-assembly offers new opportunities for optoelectronic applications, by assembling the NCs into ordered films.

Significant research effort has gone into general selfassembly strategies for various NC systems to steer the system toward its crystalline ground state. The most widespread strategies are drop-casting on a substrate, ${ }^{6}$ assembly at the air/ liquid interface, ${ }^{7}$ and casting onto a patterned template. ${ }^{8}$ This way, many superstructures have been obtained for CdSe, $\mathrm{Fe}_{3} \mathrm{O}_{4}, \mathrm{Au}$, and other nanoparticles, like superballs (SBs), supercubes, rhombic dodecahedral, octahedral, and hexapodshaped SCs. ${ }^{9-13}$ However, while their assembly could be wellcontrolled, these materials are poorly suited for photovoltaic applications due to a bandgap mismatch with solar cells.

Recently, inorganic perovskite NCs have emerged as a promising material for photovoltaic films, ${ }^{14}$ exhibiting excellent optoelectronic properties and featuring bandgap tunability by size and composition, ${ }^{15}$ energy transfer, ${ }^{16}$ and carrier multiplication capabilities. ${ }^{17}$ Yet, currently, only a few works have reported SCs made from perovskite NCs. A method that has been used to prepare $\mathrm{Cs} \mathrm{PbBr}_{3}$ SCs is tip sonication, ${ }^{14}$ with the produced SCs displaying red-shifted emission due to the interparticle electronic coupling. Microscale supercubes have been obtained by slow drying of drop-casted $\mathrm{CsPbBr}_{3} \mathrm{NCs}^{15}$ The resulting ordered superstructures showed superfluorescence such as accelerated radiative decay, photon bunching, and delayed emission pulses at high excitation density. However, all these efforts remain small scale and of rather poor external control in terms of size, morphology, and crystal quality of the superstructures, leaving the controlled large-scale assembly for applications a major challenge. As is well-known, the structural quality of the films, including morphology, crystalline degree, and order, directly affects their functionality and efficiency. Furthermore, the critical issue of the above methods is the merging of individual perovskite SCs in a few days resulting in photonic degradation driven by the detachment of the surface ligands. Therefore, finding a new solution that keeps high stability while at the same time allowing for control of the crystal quality of the perovskite SC film is an urgent task.

Received: May 11, 2020

Revised: July 23, 2020

Published: July 23, 2020 


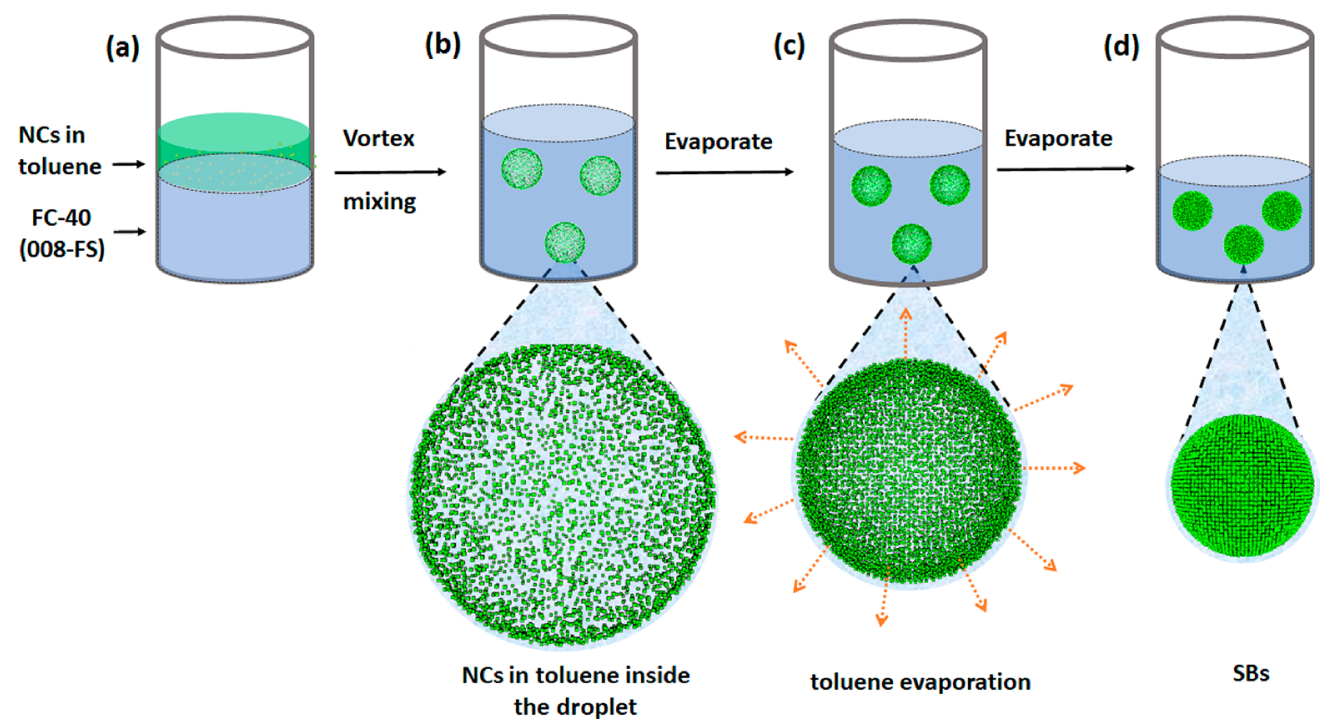

Figure 1. Schematic representation of the SCs synthesis using dual-phase emulsion assembly. (a) $\mathrm{CsPbBr}_{3} \mathrm{NCs}$ in toluene (upper) and FC-40 with 008-FS as surfactant (lower) before being vortexed. (b) Emulsion of CsPbBr 3 NCs in toluene and FC-40. The enlarged part in (b) represents the droplet of $\mathrm{CsPbBr}_{3} \mathrm{NCs}$ in toluene well-dispersed in FC-40. (c) The process of solvent evaporation. The enlarged part represents the gradual solvent evaporation followed by the shrink of the droplet. (d) After the complete evaporation of solvent, SBs form as shown in the enlarged image.

For colloidal nanoparticles, emulsion templating has been shown to provide $3 \mathrm{D}$ superstructures with exquisite order on a large scale. ${ }^{9-11}$ This technique typically uses oil-in-water emulsions with NCs dispersed in oil droplets, which crystallize upon slow evaporation of the oil. Since the evaporation rate can be very well-controlled by temperature, surfactants, or pressure, this method offers a powerful route to control the assembly and produce highly ordered superstructures in large quantities. Unfortunately, the presence of water in a typical emulsion templating process prohibits the use of perovskite NCs in this facile controlled assembly process.

Here, we present a well-controlled technique to assemble perovskite NCs into SCs and enable a high stability of the SC films using oil-in-oil emulsion templating. We solve the crucial problem of avoiding water usage by fabricating emulsions made of hydrophobic solvent-in-fluorinated oil (e.g., FC-40) stabilized by fluorinated surfactant and elucidate the effect of surfactant concentration, evaporation temperature, and cubic NC shape on the topology of the SCs. We identify an intriguing interplay between the spherical confinement of the emulsion droplets and the sharpness of the cubic NCs, in determining the structure and morphology of the final superstructure. This interplay is exploited to obtain a dense hyperstructure of SCs, arranged in a hexagonal close-packed order. The photoluminescence (PL) spectra of the SCs exhibit a redshift of $1-10 \mathrm{~nm}$ compared to the individual NCs, indicating energy transfer between the NCs. ${ }^{18}$ The resulting SCs show high morphological stability for at least two months, in contrast to previously reported assembly methods.

The formation of the SCs is illustrated in Figure 1. To obtain SCs of $\mathrm{CsPbr}_{3} \mathrm{NCs}$, emulsions of $\mathrm{NC}$ containing hydrophobic solvent in fluorinated oil are prepared by vigorous vortex mixing (Figure 1a,b). We tested different hydrophobic solvents (toluene, hexane, pentane, cyclohexane, octane) with the fluorinated oils FC-40 and HFE-7500 with surfactant (008FS) concentration in the range of $0.1-5 \mathrm{wt} \%$ for emulsion stability at room temperature (Figures S1-S3, Table S1) and found that toluene and cyclohexane formed stable emulsions with FC-40; these stable emulsions were continuously stirred and left to evaporate (Figure 1c). Upon evaporation, the emulsion droplets shrink, causing densification and selfassembly of the NCs inside, producing highly ordered SCs (Figure 1d). The final SC suspension was centrifugated and dispersed into FC-40 with 008-FS as surfactant and then dropcast onto transmission electron microscopy (TEM) grids for imaging. Two different batches of $\mathrm{CsPBr}_{3} \mathrm{NCs}$ synthesized by hot-injection reaction were used: one freshly synthesized and one six months after synthesis. These have average sizes of 10 and $14 \mathrm{~nm}$, respectively, and differ in the sharpness of the cubic shape, as determined by scanning transmission electron microscopy (STEM). Both exhibit the expected pure phase of orthorhombic $\mathrm{Cs} \mathrm{PbBr}_{3}$, as well as the expected absorption onset at $498 \mathrm{~nm}(2.49 \mathrm{eV})$ and $499 \mathrm{~nm}(2.48 \mathrm{eV})$, respectively, and the PL peak position at $519 \mathrm{~nm}(2.39 \mathrm{eV}) .{ }^{19}$ The full sample details are given in Figure S4.

STEM images of the superstructures obtained from aged $\mathrm{NCs}$ by evaporation at $20{ }^{\circ} \mathrm{C}$ reveal highly ordered SBs, as shown in Figure $2 a-c$. Lattice fringes distinguished for the individual NCs show their atomic lattice alignment. The SBs exhibit a broad size distribution with the average size of $\sim 90$ $\mathrm{nm}$, as shown in Figure $2 \mathrm{~b}$. The high degree of ordering is reminiscent of a previous assembly of CdSe $\mathrm{NC} \mathrm{SBs;}{ }^{11}$ however, these perovskite NCs exhibit a cubic shape, resulting in a cubic instead of a face-centered cubic ordering. When the aliphatic ligands on the $\mathrm{CsPbBr}_{3} \mathrm{NCs}$ are intermixed with solvent molecules, their steric stabilization prevents interparticle agglomeration and confers colloidal stability on the NCs. $^{20}$ For the CdSe NCs in oil-in-water emulsions, we observed that their assembly is determined by effective hardsphere behavior, while the late stages are accompanied by an additional attractive component upon compression of the ligand shell due to the receding interface of the evaporating droplets. ${ }^{11}$ We speculate that, in analogy, the perovskite NC assembly may be dominated by an effective hard (rounded) cube behavior. $^{21-23}$

The morphology of the superstructures changes from spherical to cubic when we use freshly synthesized NCs exhibiting sharp edges; a single supercube is shown in Figure 

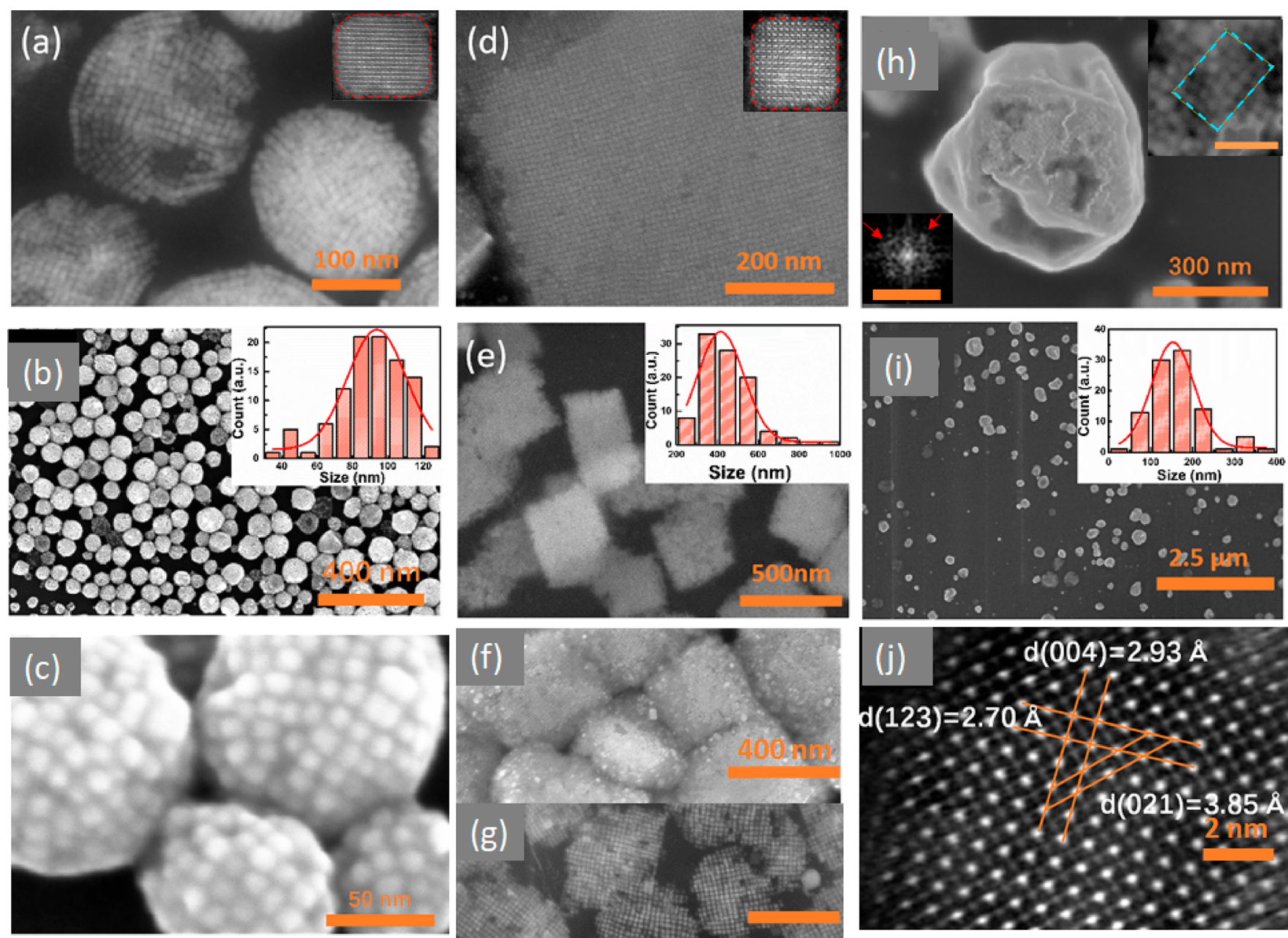

Figure 2. STEM images and size distribution of the perovskite NC superstructures. (a) SBs-90 obtained with 0.1 wt $\%$ surfactant at $20{ }^{\circ} \mathrm{C}$ assembled from aged NCs. (inset) HRTEM image of a typical aged NC and fitting with a superellipse (dashed line). (b) Miniaturized image with size histogram of SBs (inset). (c) STEM image of SBs-90 with clear individual NCs. (d) Supercube obtained with 0.1 wt $\%$ surfactant at $20{ }^{\circ} \mathrm{C}$ assembled from fresh NCs. (inset) HRTEM image of a typical fresh NC and fitting with a superellipse (dashed line). (e) Miniaturized image with size histogram of the supercubes (inset). (f) Tilted STEM of supercubes. (g) SBs obtained from NCs stored for six weeks, assembled with 0.1 wt \% surfactant at $20{ }^{\circ} \mathrm{C}$. Scale bar: $400 \mathrm{~nm}$. (h) Irregular SCs assembled with $2 \mathrm{wt} \%$ surfactant at $40{ }^{\circ} \mathrm{C}$ from aged NCs. (insets) Enlarged SEM image (top right), scale bar $50 \mathrm{~nm}$, and FFT image of the selected area (bottom left), scale bar, $0.51 / \mathrm{nm}$. (i) Miniaturized image with size distribution for irregular SCs (inset). (j) HRTEM image of a single NC in an SB revealing the characteristic $\mathrm{CsPbBr}_{3}$ structure.

$2 \mathrm{~d}$, where the individual NCs are observed to exhibit nearperfect cubic ordering. These supercubes have an average size of $400 \mathrm{~nm}$ as shown in the inset of Figure 2e and Figure S5. The 3D structure of the supercubes is illustrated by tilting to different angles (Figures $2 \mathrm{f}$ and S6), clearly revealing their cubic shape. We hypothesize that, in this case, the sharper edges of the fresh NCs made the dense cubic superstructure more stable against the pressure of the receding spherical droplet interface so that it maintained its cubic morphology.

This trend is further confirmed when we repeat the assembly after six weeks; the gradual loss of sharp edges resulted in a gradual transition from regular to rounded and irregular SCs (Figure 2g and Figure S7). We thus find a systematic dependence of the superstructure morphology on the sharpness of the cubic building blocks. This dependence is in qualitative agreement with previous work on colloidal cubes reporting a strong effect of the sharpness of the cubic particles on their ordering. ${ }^{21,22}$ We find that even the morphology of the superstructure is affected by this interplay, possibly due to the smaller NC size and lower interfacial tension: the measured interfacial tension of the oil-in-fluorinated (FC-40) oil emulsion containing $0.1 \mathrm{wt} \%$ surfactant is $\sim 3.54 \mathrm{mN} / \mathrm{m}$ (Figure S8), while that for toluene/water is $\sim 18 \mathrm{mN} / \mathrm{m},{ }^{24}$ a factor of 5 smaller. Furthermore, we characterize the sharpness of the cubic NCs by fitting their apparent two-dimensional (2D) shape in STEM images to the equation of a superellipse $\left|\frac{x}{a}\right|^{m}+\left|\frac{y}{b}\right|^{m} \leq 1$, where $x$ and $y$ are the coordinates along the superellipse, $a$ and $b$ are the semi-axes of the superellipse (or equivalently half the side lengths of the rounded rectangle), and $m$ is the shape parameter ( $m=2$ for an ellipse, and $m \rightarrow$ $\infty$ for a rectangle). ${ }^{23}$ Fitting $\sim 100$ NCs, we find average shape parameters of $m=5.3$ for the freshly synthesized and $m=3.6$ for the aged sample (see the insets of Figure 2a,d, and Figure S9), corresponding to a sharper and more rounded NC shape, respectively. The sharper cubic shape, together with the low surface tension, makes the assembled structure more resistant against the spherical interface.

The interplay of interfacial tension and NC shape is further explored by lowering the interfacial tension. Increasing the surfactant concentration from 0.1 to $2 \mathrm{wt} \%$ results in a 
(a)

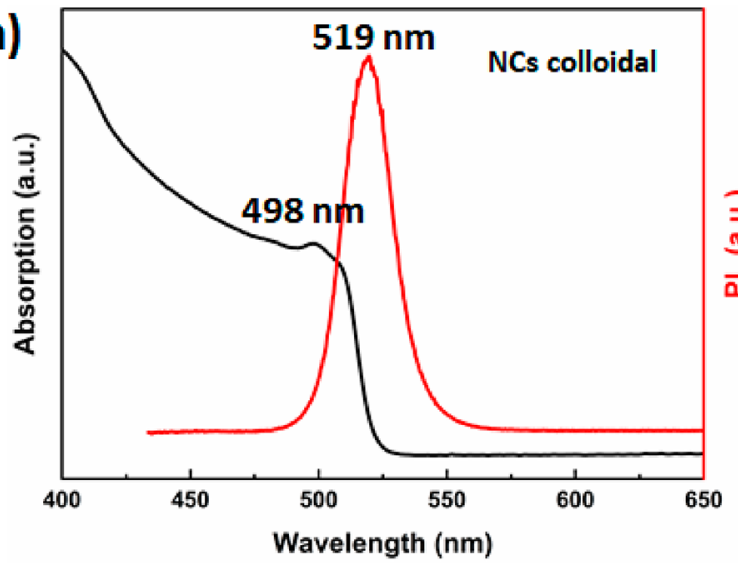

(c)

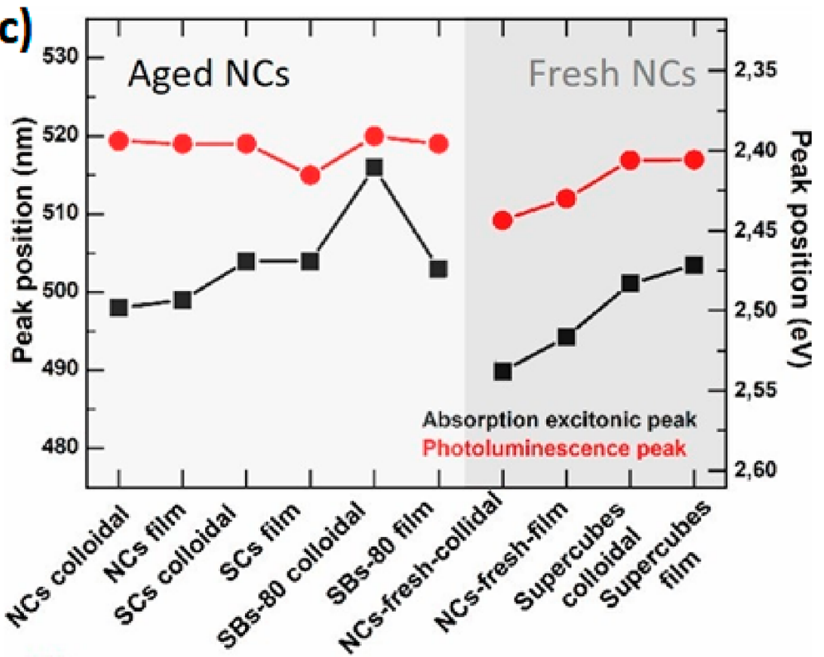

(b)

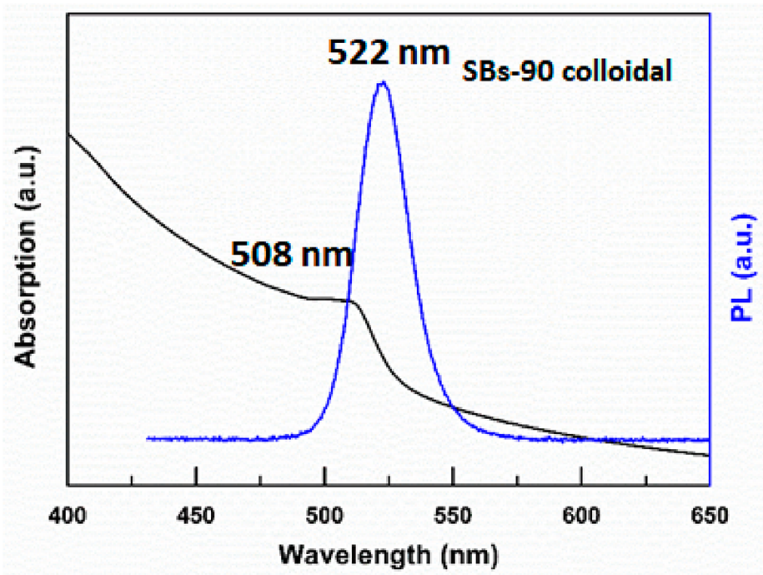

(d)

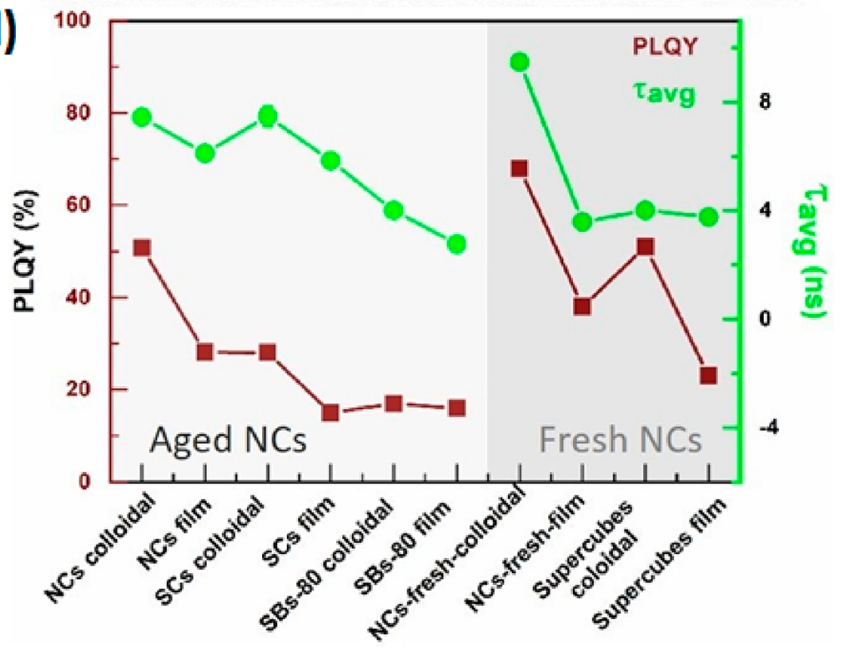

Figure 3. Optical properties of the NCs and superstructures. (a) UV-Visible absorption and PL spectra of colloidal CsPbBr $\mathrm{NCs}_{3} \mathrm{NC}$. (b) UV-Visible absorption and PL spectra of colloidal superballs (SBs-90) assembled from aged NCs. (c) Absorption and PL peak position of the perovskite NCs, irregular SCs, SBs assembled from old NCs, supercubes (suspended and drop-casted). (d) Corresponding PL QY and average lifetime for NCs and SCs.

wrinkled morphology (Figure 2h,i), while the NCs still exhibit cubic ordering (see insets of Figure $2 \mathrm{~h}$ and Figure S10). This morphology suggests that, upon drying, the surface tension was no longer able to maintain a regular interface. The surfactant concentration also affects the superstructure size: higher surfactant concentrations lead to smaller droplets and thus to smaller superstructures with an average size of $\sim 150 \mathrm{~nm}$, in contrast to the average size of $420 \mathrm{~nm}$ (SBs-420 in Figures S11 and S12). This might be a direct consequence of the lower interfacial tension, allowing for smaller droplets with higher curvature. We also note that, when lowering the evaporation speed, we obtain much higher quality supercrystals, consistent with previous work on CdSe superstructures, where a highquality crystal (67\% crystallinity, i.e., $67 \%$ of all superballs exhibiting crystalline order) was observed at a slow evaporation rate, and a rather amorphous structure was seen for faster quench rates. $^{11,25}$ In all of the above cases, the phase of the individual NCs is preserved as shown by representative highresolution transmission electron microscopy (HRTEM) images of an NC inside a superstructure in Figure $2 \mathrm{j}$. We note that, depending on the preparation protocol, other specific morphologies like vesicles or hollow "donuts" are also obtained (Figure S12); these could play a role in potential heterogeneous catalysis ${ }^{26}$ as well as protecting, carrying, and delivering cargo. ${ }^{27}$
The presented emulsion-templated assembly method provides a well-controlled alternative to the few existing perovskite NC assembly methods based on drop-casting or post-treatments of the perovskite NCs by specific solvents, for example, methyl acetate. ${ }^{2,3,14,15}$ These works related to the assembly of NCs face several challenges. (1) The SCs are only stable for approximately two weeks due to the detachment of surface ligands. $^{15}$ (2) The volume ratio between the NC's solvent and the solvent applied for treatment should be precisely controlled to obtain a specific morphology. ${ }^{15}$ (3) Pretreatments of NCs sometimes are necessary to make them more hydrophilic to the substrates. ${ }^{2}$ (4) Irregular morphology exists as well as individual NCs or bulklike impurities in the supercrystals. $^{3}$ In contrast, the oil-in-oil emulsion template presented here provides an easily controllable bulk assembly method that naturally supports upscaling. Choosing the fluorinated oil, immiscible with the hydrophobic solvent dispersing the NCs, as continuous phase results in the improved control over the assembly of the perovskite NCs. By varying the assembly conditions, we can obtain distinct superstructure morphologies such as SBs, supercubes, and superhexagons compared to conventional methods resulting only in supercubes and disordered superstructures.

The optical properties of the resulting SCs and NCs, both in colloidal form and films, are shown in Figure 3. In all cases, we 
(a)

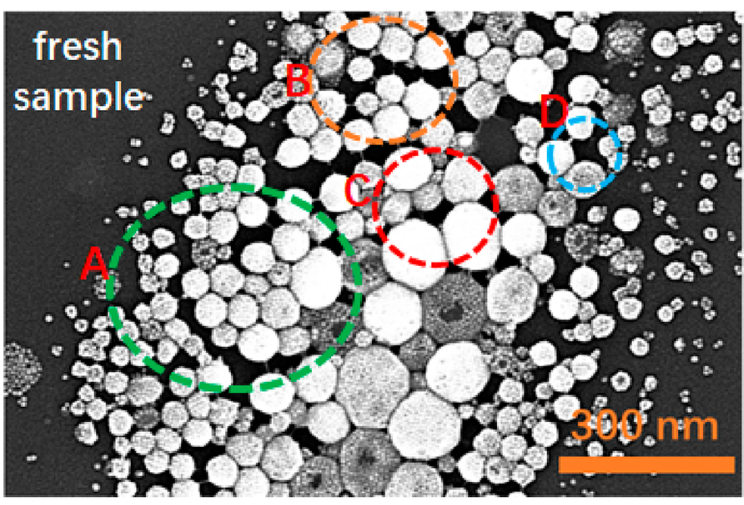

(c)

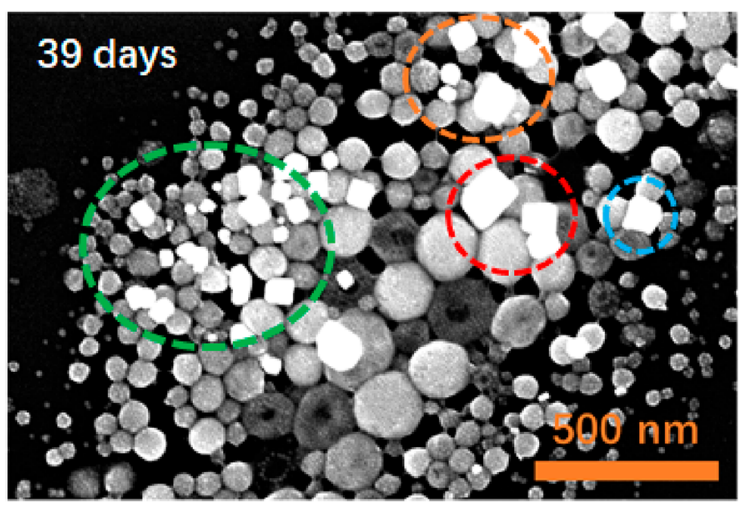

(b)

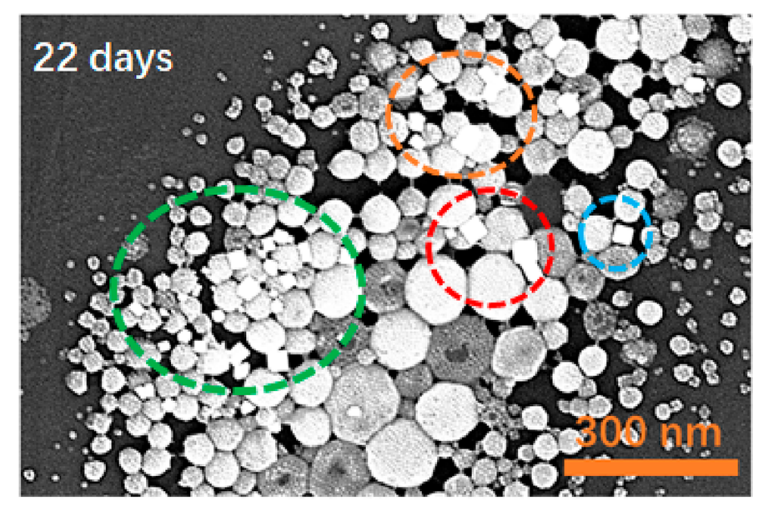

(d)

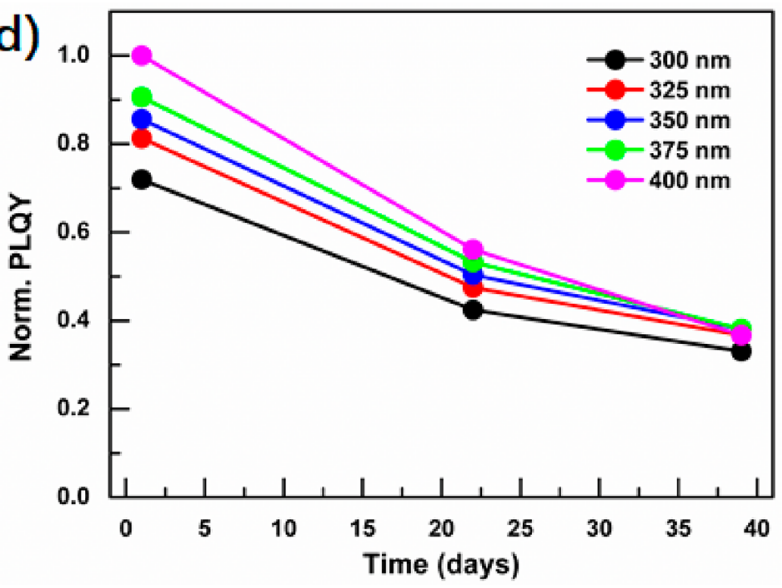

Figure 4. Morphological and optical stability of the superstructures. STEM images at the same position on the TEM grid for (a) fresh sample and after (b) 22 and (c) $39 \mathrm{~d}$ of deposition, stored in air. (d) Wavelength-dependent PL QY for SBs film after stored at ambient condition.

observe a weak red shift $(1-10 \mathrm{~nm})$ compared to the individual NCs, in agreement with previous work on perovskite NC superstructures, demonstrating energy transfer between the NCs. ${ }^{2,3}$ In Figure 3a,b, we show that the peaks in the absorption and PL spectra shift from 498 and $519 \mathrm{~nm}$ for NCs, respectively, to 508 and $522 \mathrm{~nm}$ for SBs after assembling the colloidal NCs into SBs. This red shift is most pronounced for the supercubes made from fresh NCs, exhibiting a PL red shift of $10 \mathrm{~nm}$ with respect to the dispersed NCs (see Figure 3c). For the SBs made from aged NCs, the absorption onset continuously red-shifts from the dispersed NCs via the irregular SCs to the large SBs, indicating the availability of lower states in the increasingly dense and ordered structures. These signatures of increasing coupling of the NCs are supported by the measurement of the photoluminescence quantum yield (PL QY) and lifetime (Figure 3d). For both NC samples, we find a systematic decrease of both the PL QY and the lifetime, indicating increasing nonradiative relaxation due to the increasing delocalization and increasing energy transfer. The PL QY decreases from $50.8 \%$ to $28.2 \%$ from the NC suspension to film, which further decreases to $28 \%$ and $15 \%$, respectively, upon assembling NCs into irregular superstructures, in solution and drop-casted, which further decreases to values of $\sim 17 \%$ for densely packed SBs. This trend is qualitatively mirrored in the PL lifetime, which shows a continuous decrease from the irregular superstructures to the densely packed SBs, as shown in Figure 3d, and supported by full time-dependent data in Figure S13. The decrease of the PL $\mathrm{QY}$ and reduction of the lifetime indicate significant energy transfer between the assembled NCs, which becomes the most pronounced for the ordered, dense SBs. The fresh NCs show an even more abrupt trend. Their PL QY and lifetime start at higher values, as expected for the better NC quality, and decay rapidly upon assembly into films and supercubes, as expected from their higher degree of order and coupling, and are consistent with their stronger PL redshift. The detailed values are reported in the Supporting Information, Tables S2-S4. These results highlight the direct control of the optoelectronic properties by the structural control afforded by the emulsiontemplated assembly technique.

Most importantly, we observe a highly increased stability of the NCs inside the superstructures. We tested the stability of the assembled SCs both in terms of their morphology and their optical properties by repeated imaging by STEM and by measurement of the PL QY, see Figure 4. The SCs assembled from individual NCs are still well-preserved without degradation after 22 and even $39 \mathrm{~d}$ (Figure $4 \mathrm{a}-\mathrm{c}$ ), without merging of the NCs inside. In addition, small cubes appeared that grew into bigger cubes (Figure 4c), which, by detailed chemical composition analysis by energy-dispersive X-ray (EDX), we identified to consist of the same perovskite material. These cubes were not observed at any other sample position after being exposed to ambient condition, even after two months, suggesting that their appearance is induced by the electron beam illumination upon imaging; see Supporting Information, Figures S14 and S15 for full details. This is different from our previous work, where we rather observed the electron-beam irradiation to enhance the stability of NCs. ${ }^{28}$ The good stability of the SCs is also reflected in their optical properties, as shown in Figure 4d. After $39 \mathrm{~d}$ at ambient conditions, $\sim 45 \%$ 
of the PL QY is preserved for all measurements. These results are in stark contrast to the rather rapid decay of morphology and PL reported previously for perovskite cuboidal superstructures, which merged into single nanoplatelets within two weeks. ${ }^{15}$ We hypothesize that the FC-40 oil plays a crucial role, being an inert oil resistant to high-temperature degradation. After the complete drying of the toluene phase, the resulting SCs are stabilized by the 008-FS, hindering ligand detachment, as the ligands are insoluble in the fluorinated oil, and avoiding further degradation and merging. Additionally, merging into fused cubes can be induced on purpose by electron-beam irradiation and chemical treatments to enhance the performance of devices.

We finally explore the emulsion droplet technique for the hierarchical assembly of dense films. The idea is to use the superstructures themselves as building blocks for the assembly on a larger scale. Indeed, the control over size and morphology of the superstructures can be exploited to tune them for assembly into dense ordered layers, hyperstructures of an ordered arrangement of SCs. To explore this route, we deposit the emulsion with the NC-loaded droplets onto a substrate (TEM grid) in the wet state, before they are completely dry, after evaporating for $48 \mathrm{~h}$ (complete drying takes $72 \mathrm{~h}$ and yields SBs). The resulting deposits exhibit hierarchical ordering as shown in Figure 5. Hexagonal-shaped superstructures

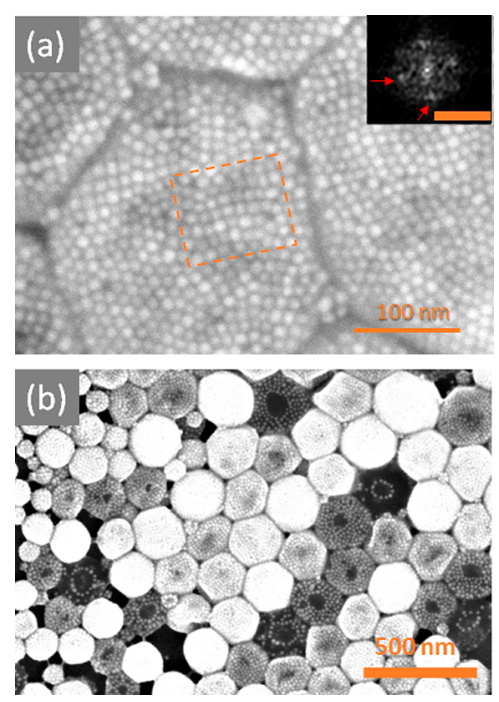

Figure 5. STEM images of the perovskite NC superstructures on TEM grids. (a) Superhexagons obtained by drop-casting the emulsion (with 0.1 wt \% surfactant) on the TEM grid before complete drying, after evaporation of $48 \mathrm{~h}$ at $20{ }^{\circ} \mathrm{C}$. (inset) The FFT image for the selected area. Scale bar: $0.51 / \mathrm{nm}$. (b) Miniaturized image showing the ordered packed superhexagons.

arrange into a hexagonal close-packed lattice, while preserving the cubic or hexagonal ordering of the NCs inside. As shown in Figure S16, the superhexagons appear in the center of the deposited droplet, while single superballs are found along the outer edge of the structure. Upon deposition of more material (larger or more drops on the substrate), the hyperstructure will cover larger areas. Another factor is the polydispersity of the starting droplets leading to size-sorting during the drying step. $^{29,30}$ This can be addressed by using microfluidics to generate uniform droplets. From the FFT images of the selected area, as shown in the inset of Figure 5a and Figure S17, crystalline ordering can be identified from the appearance of four spots, indicating domains of cubic ordering in the superhexagons. These superhexagons are arranged in a hexagonal order again, affording the possibility for densely packed films for potential devices. Apparently, the "soft" emulsion droplets after deposition on the substrate were sufficiently stable to form a hexagonal lattice, which further densified upon drying. This way, close-packed perovskite films of superhexagons can be obtained. The thickness of the film is on the order of $100 \mathrm{~nm}$, which is the right order of magnitude for optoelectronic applications. ${ }^{31}$ This type of hierarchical ordering could be used to cover larger areas by a simple upscalable process. Furthermore, the application of microfluidic techniques would allow for monodisperse droplets and highly ordered hyperstructures.

We have demonstrated the controlled assembly of stable inorganic perovskite NC superstructures using emulsion templating based on oil-in-fluorinated oil emulsions, yielding supercubes, SBs, and superhexagons. The morphology of the superstructures results from a subtle interplay of roundness of the cubic NCs and surface tension of the spherical confinement. This interplay can be exploited to obtain hyperstructures, hexagonal close-packed arrangements of superhexagons. These results open new directions for the hierarchical self-assembly of high-quality, stable, and homogeneous films, offering new opportunities for phosphor layers and efficient perovskite NC-based devices after chemical or electron irradiation treatments, which has been realized in CdSe supercrystals. ${ }^{32}$ In particular, the choice of a hydrophobic liquid, toluene, in a fluorinated oil (FC-40) droplet allows for the existing expertise garnered by research in microfluidic technologies to apply this approach in a rapid and timely manner to inorganic perovskite NC assembly. ${ }^{33}$

\section{ASSOCIATED CONTENT}

\section{Supporting Information}

The Supporting Information is available free of charge at https://pubs.acs.org/doi/10.1021/acs.nanolett.0c02005.

The synthesis process, characterization methods, stability of the emulsion system, XRD, STEM, HRTEM images, quantification modeling, surface tension and optical measurements (UV, PL, PL QY, lifetime), additional analysis and discussions (PDF)

\section{AUTHOR INFORMATION}

\section{Corresponding Author}

Peter Schall - Institute of Physics, University of Amsterdam, 1098 XH Amsterdam, The Netherlands; Email: P.Schall@ uva.nl

\section{Authors}

Yingying Tang - Institute of Physics, University of Amsterdam, 1098 XH Amsterdam, The Netherlands; 으이. orid.org/00000003-4603-9499

Leyre Gomez - Institute of Physics, University of Amsterdam, 1098 XH Amsterdam, The Netherlands; Catalan Institute of Nanoscience and Nanotechnology, CSIC, BIST, and CIBERBBN, 08193 Bellaterra Barcelona, Spain

Arnon Lesage - Institute of Physics, University of Amsterdam, 1098 XH Amsterdam, The Netherlands; (ㅇ orcid.org/00000002-3102-3980 
Emanuele Marino - Department of Chemistry, University of Pennsylvania, 19104-6323 Philadelphia, Pennsylvania, United States; (1) orcid.org/0000-0002-0793-9796

Thomas E. Kodger - Physical Chemistry and Soft Matter, Wageningen University \& Research, 6708 WE Wageningen, The Netherlands

Janne-Mieke Meijer - Institute of Physics, University of Amsterdam, 1098 XH Amsterdam, The Netherlands

Paul Kolpakov - Institute of Physics, University of Amsterdam, 1098 XH Amsterdam, The Netherlands

Jie Meng - Department of Chemistry, Technical University of Denmark, DK-2800 Kongens Lyngby, Denmark; 이이.org/ 0000-0002-3813-5221

Kaibo Zheng - Department of Chemistry, Technical University of Denmark, DK-2800 Kongens Lyngby, Denmark; Department of Chemical Physics and NanoLund, Lund University, 22100 Lund, Sweden

$\zeta_{\text {Tom Gregorkiewicz - Institute of Physics, University of }}$ Amsterdam, 1098 XH Amsterdam, The Netherlands; (ㄱ) orcid.org/0000-0003-2092-8378

Complete contact information is available at: https://pubs.acs.org/10.1021/acs.nanolett.0c02005

\section{Author Contributions}

Y.T., L.G., E.M., T.K., T.G., and P.S. conceived the project and designed the experiments. Y.T. prepared the samples and analyzed the data. A.L. helped with the optical measurements and analyzed the data. J.-M.M. and Y.T. performed the modeling of the sharpness of the materials. P.K. performed the surface tension experiment. J.M. and K.B.Z. performed the HRTEM measurements. All authors discussed the results and their interpretation.

Notes

The authors declare no competing financial interest.

${ }^{\zeta}$ The author passed away on the seventh of July, 2019.

\section{ACKNOWLEDGMENTS}

We are grateful for the Dutch Technology Foundation STW, The Netherlands Organization for Scientific Research (NWO), and the Joint Solar Program (JSP III, 680-91-011) of The NWO for financial support. We acknowledge P. Koppejan, $\mathrm{H}$. Agrawal, M. van der Laan, C.-C. Huang, H. R. de Latour, E. Zheng, and D. van der Gon for their kind help.

\section{REFERENCES}

(1) Kagan, C. R.; Murray, C. B. Charge transport in strongly coupled quantum dot solids. Nat. Nanotechnol. 2015, 10, 1013-1026.

(2) Rainò, G.; Becker, M. A.; Bodnarchuk, M. I.; Mahrt, R. F.; Kovalenko, M. V.; Stöferle, T. Superfluorescence from lead halide perovskite quantum dot superlattices. Nature 2018, 563, 671-675.

(3) Baranov, D.; Toso, S.; Imran, M.; Manna, L. Investigation into the photoluminescence red shift in cesium lead bromide nanocrystal superlattices. J. Phys. Chem. Lett. 2019, 10, 655-660.

(4) Lan, X. Z.; Chen, M. L.; Hudson, M. H.; Kamysbayev, V.; Wang, Y. Y.; Guyot-Sionnest, P.; Talapin, D. V. Quantum dot solids showing state-resolved band-like transport. Nat. Mater. 2020, 19, 323-329.

(5) Henzie, J.; Grünwald, M.; Widmer-Cooper, A.; Geissler, P. L.; Yang, P. D. Self-assembly of uniform polyhedral silver nanocrystals into densest packings and exotic superlattices. Nat. Mater. 2012, 11, 131-137.

(6) Rabani, E.; Reichman, D. R.; Geissler, P. L.; Brus, L. E. Dryingmediated self-assembly of nanoparticles. Nature 2003, 426, 271-274.
(7) Dong, A.; Chen, J.; Vora, P. M.; Kikkawa, J. M.; Murray, C. B. Binary nanocrystal superlattice membranes self-assembled at the liquid-air interface. Nature 2010, 466, 474-477.

(8) Saboktakin, M.; Ye, X. C.; Chettiar, U. K.; Engheta, N.; Murray, C. B.; Kagan, C. R. Plasmonic enhancement of nanophosphor upconversion luminescence in Au nanohole arrays. ACS Nano 2013, 7, 7186-7192.

(9) de Nijs, B.; Dussi, S.; Smallenburg, F.; Meeldijk, J. D.; Groenendijk, D. J.; Filion, L.; Imhof, A.; van Blaaderen, A.; Dijkstra, M. Entropy-driven formation of large icosahedral colloidal clusters by spherical confinement. Nat. Mater. 2015, 14, 56-60.

(10) Vanmaekelbergh, D.; van Vugt, L. K.; Bakker, H. E.; Rabouw, F. T.; de Nijs, B.; van Dijk-Moes, R. J. A.; van Huis, M. A.; Baesjou, P. J.; van Blaaderen, A. Shape-dependent multiexciton emission and whispering gallery modes in supraparticles of $\mathrm{CdSe} /$ multishell quantum dots. ACS Nano 2015, 9, 3942-3950.

(11) Marino, E.; Kodger, T. E.; Wegdam, G. H.; Schall, P. Revealing driving forces in quantum dot supercrystal assembly. Adv. Mater. 2018, 30, 1803433.

(12) Dreyer, A.; Feld, A.; Kornowski, A.; Yilmaz, E. D.; Noei, H.; Meyer, A.; Krekeler, T.; Jiao, C. G.; Stierle, A.; Abetz, V.; Weller, H.; Schneider, G. A. Organically linked iron oxide nanoparticle SCs with exceptional isotropic mechanical properties. Nat. Mater. 2016, 15, $522-528$.

(13) Ushakova, E. V.; Cherevkov, S. A.; Volgina, D. -O. A.; Zakharov, V. V.; Komissarenko, F. E.; Shcherbakov, A. A.; Hogan, B. T.; Baldycheva, A.; Fedorov, A. V.; Nabiev, I. R.; Baranov, A. V. From colloidal CdSe quantum dots to microscale optically anisotropic SCs through bottom-up self-assembly. J. Mater. Chem. C 2018, 6, 1290412911.

(14) Tong, Y.; Yao, E.-P.; Manzi, A.; Bladt, E.; Wang, K.; Döblinger, M.; Bals, S.; Müller-Buschbaum, P.; Urban, A. S.; Polavarapu, L.; Feldmann, J. Spontaneous self-assembly of perovskite nanocrystals into electronically coupled SCs: toward filling the green gap. $A d v$. Mater. 2018, 30, 1801117.

(15) van der Burgt, J. S.; Geuchies, J. J.; van der Meer, B.; Vanrompay, H.; Zanaga, D.; Zhang, Y.; Albrecht, W.; Petukhov, A. V.; Filion, L.; Bals, S.; Swart, I.; Vanmaekelbergh, D. Cuboidal supraparticles self-assembled from cubic $\mathrm{Cs}_{\mathrm{PbBr}}$ perovskite nanocrystals. J. Phys. Chem. C 2018, 122, 15706-15712.

(16) Ghimire, S.; Takahashi, K.; Takano, Y.; Nakamura, T.; Biju, V. Photon recycling by energy transfer in piezochemically synthesized and close-packed methylammonium lead halide perovskites. J. Phys. Chem. C 2019, 123, 27752-27758.

(17) de Weerd, C.; Gomez, L.; Capretti, A.; Lebrun, D. M.; Matsubara, E.; Lin, J. H.; Ashida, M.; Spoor, F. C. M.; Siebbeles, L. D. A.; Houtepen, A. J.; Suenaga, K.; Fujiwara, Y.; Gregorkiewicz, T. Efficient carrier multiplication in $\mathrm{CsPb}_{3}$ perovskite nanocrystals. Nat. Commun. 2018, 9, 4199.

(18) de Weerd, C.; Gomez, L.; Zhang, H.; Buma, W. J.; Nedelcu, G.; Kovalenko, M. V.; Gregorkiewicz, T. Energy transfer between inorganic perovskite nanocrystals. J. Phys. Chem. C 2016, 120, 13310-13315.

(19) Lin, J. H.; Gomez, L.; de Weerd, C.; Fujiwara, Y.; Gregorkiewicz, T.; Suenaga, K. Nano Lett. 2016, 16, 7198-7202.

(20) Kim, D.; Lee, D. C. Surface ligands as permeation barrier in the growth and assembly of anisotropic semiconductor nanocrystals. J. Phys. Chem. Lett. 2020, 11, 2647-2657.

(21) Wang, D.; Hermes, M.; Kotni, R.; Wu, Y. T.; Tasios, N.; Liu, Y.; de Nijs, B.; van der Wee, E. B.; Murray, C. B.; Dijkstra, M.; van Blaaderen, A. Interplay between spherical confinement and particle shape on the self-assembly of rounded cubes. Nat. Commun. 2018, 9, 2228.

(22) Rossi, L.; Soni, V.; Ashton, D. J.; Pine, D. J.; Philipse, A. P.; Chaikin, P. M.; Dijkstra, M.; Sacanna, S.; Irvine, W. T. M. Shapesensitive crystallization in colloidal superball fluids. Proc. Natl. Acad. Sci. U. S. A. 2015, 112, 5286-5290. 
(23) Meijer, J.-M.; Pal, A.; Ouhajji, S.; Lekkerkerker, H. N.W.; Philipse, A. P.; Petukhov, A. V. Observation of solid-solid transitions in 3D crystals of colloidal superballs. Nat. Commun. 2017, 8, 14352.

(24) Bąk, A.; Podgórska, W. Interfacial and surface tensions of toluene/water and air/water systems with nonionic surfactants Tween 20 and Tween 80. Colloids Surf., A 2016, 504, 414-425.

(25) Marino, E.; Keller, A. W.; An, D.; van Dongen, S.; Kodger, T. E.; MacArthur, K. E.; Heggen, M.; Kagan, C. R.; Murray, C. B.; Schall, $\mathrm{P}$. Favoring the growth of high-quality, three-dimensional supercrystals of nanocrystals. J. Phys. Chem. C 2020, 124, 11256-11264.

(26) Tricot, Y. M.; Fendler, J. H. Colloidal catalyst-coated semiconductors in surfactant vesicles: in situ generation of rhodium-coated cadmium sulfide particles in dihexadecyl phosphate vesicles and their utilization for photosensitized charge separation and hydrogen generation. J. Am. Chem. Soc. 1984, 106, 7359-7366.

(27) Paczkowski, J. E.; Richardson, B. C.; Fromme, J. C. Cargo adaptors: structures illuminate mechanisms regulating vesicle biogenesis. Trends Cell Biol. 2015, 25, 408-416.

(28) Gomez, L.; Lin, J. H.; De Weerd, C.; Poirier, L.; Boehme, S. C.; Von Hauff, E.; Fujiwara, Y.; Suenaga, K.; Gregorkiewicz, T. Extraordinary interfacial stitching between single all-inorganic perovskite nanocrystals. ACS Appl. Mater. Interfaces 2018, 10, 5984-5991.

(29) Utada, A. S.; Lorenceau, E.; Link, D. R.; Kaplan, P. D.; Stone, H. A.; Weitz, D. A. Monodisperse double emulsions generated from a microcapillary device. Science 2005, 308, 537-541.

(30) Zhu, P. G.; Wang, L. Q. Passive and active droplet generation with microfluidics: a review. Lab Chip 2017, 17, 34-75.

(31) Liu, D. Y.; Gangishetty, M. K.; Kelly, T. L. Effect of $\mathrm{CH}_{3} \mathrm{NH}_{3} \mathrm{PbI}_{3}$ thickness on device efficiency in planar heterojunction perovskite solar cells. J. Mater. Chem. A 2014, 2, 19873-19881.

(32) Peng, Y. L.; Song, G. S.; Hu, X. H.; He, G. J.; Chen, Z. G.; Xu, X. F.; Hu, J. Q. In situ synthesis of P3HT-capped CdSe superstructures and their application in solar cells. Nanoscale Res. Lett. 2013, 8, 106.

(33) Bodnarchuk, M. I.; Li, L.; Fok, A.; Nachtergaele, S.; Ismagilov, R. F.; Talapin, D. V. Three-dimensional nanocrystal superlattices grown in nanoliter microfluidic plugs. J. Am. Chem. Soc. 2011, 133, $8956-8960$. 